Eisenjodür von Tinct. ferri jodat. u. Tinct. ferri muriat. 261

Tinct. ferri mur., eins der wirksamslen Eisenmittel, ist durch den Umstand, dass man ihrer Haltbarkeit, d. h. dem zersetzenden Einfluss des atmophärischen Sauerstoffs nicht begegnete, die Ansäuerung durch wenig Salzsäure unterliess, völlig in Misscredit gesetzt. Schon vor vielen Jahren habe ich im Archiv der Pharnacie darauf hingewiesen, und die hiesigen Herren Aerzte bedienen sich unausgesetzt und, wie ich höre, mit den glänzendsten Erfolgen dieser alten ehrwürdigen Eisentinctur, die so hallbar wie das Eisen selbst ist. Dieselbe wird in hiesiger Offcin wie folgt dargestellt: Auf 1 Unze reclificirten Weingeist wird 1 Drachme Eisenchlorür, das, wenn es auch basiscb, durch einiges Oxychlorür gelbgefärbt ausfällt, in eine weisse Glasflasche gethan, dem einige Salzsäure beigegeben ist, den Sonnenstrahlen bis zur völligen Farblosigkeit ausgesetzt. Von dem etwa sich gebildet habenden gelben Niederschlage vorsichlig abgegossen, wird der Tinctur auf die Unze $\frac{2}{2}$ Scrupel Salzsäure zugefügt. Dieses Millel ist zu Anfang seiner Darstellung farblos, wird dann grün und endlich gelb von Farbe, hat einen höchst angenehmen Chloräthylgeruch und wird von Zeit zu Zeit den Sonnenstrahlen preisgegeben, dem Lichte ausgesetzt aufbewahrt.

\title{
leber Tinctura ferri acetici aetherea und die eigentliche chemische Natur des officinellen essigsauren Eisenliquors;
}

\author{
H. B e cker, \\ Apotheker in Essen.
}

Lu den Präparaten, die dem Pharmaceuten, wenn er sie stets in untadelhafter Beschaffenheit verabreichen will viele Schwierigkeiten bereiten, gehört unstreitig vor Allem. die Tinct. ferri acet. aether. Es ist wohl bekannt genug, nicht allein, dass keineswegs jedes Eisenoxydhydrat sich in Essigsäure in binreichender Menge auflöst, sondern auch, 
dass die Tinctur nur zu leicht in einen Zustand von Zersetzung übergeht, welcher sie sehr bald gänzlich unbrauchbar macht.

Besonders der letztere Umstand macht bei diesem Mittel viel zu schaffen. Die Zersetzung besteht im Allgemeinen in einer Ablagerung von Eisenoxyd oder dem ähnlichen, die aber sehr verschieden schnell eintritt, und auch in sonstiger Beziehung sich in verschiedener Weise äussert. Zuweilen wird die Tinctur sehr schnell gelbtrübe und gerinnt dann meistens zu einer festen gallertartigen Masse. In andern Fällen hält sie sich dagegen länger; es erfolgt dann ein allmälig zunehmender, hellbrauner Absatz, oder auch eine suspensive Trübung, die erst nach und nach bis zum gänzlichen Verderben des Mittels fortschreitet, aber dann in der Regel nicht in ein Gelatiniren ubergeht. Worin der Absatz eigentlich bestehe, ob derselbe ein eigentliches basisches Salz, oder bloss Eisenoxyd sei, ist schwer zu ermitteln, indem der Niederschlag bei jedem Versuche, ihn zu sammeln und auszuwaschen, durch die Filter geht; wahrscheinlich ist es, wie sich später ergeben wird, ein unbestimmtes Gemenge von basischem essigsaurem Salz und freiem Eisenoxyd. Dieser Absatz erfolgt übrigens nicht minder in Flüssigkeiten, die einen Säureuiberschuss enthalten, als in ganz gesälligten Präparaten, und nicht allein in der fertig gemischten Tinctur, sondern auch in der für sich aufbewahrten essigsauren Auflösung des Eisenoxydhydrats.

Schon seit einer Reihe von Jahren hat mich diese Erscheinung und die Erforschung ihrer Ursachen beschäftigt. Nach meinen ersten Beobachtungen schien es mir, als habe die Einwirkung von Licht und Wärme den wesentlichsten Antheil daran, und dass beide Agentien unter Umständen entschieden mitwirkend seien, zeigten directe Versuche allerdings auch auf das Bestimmteste; aber die Grundursache musste doch noch eine andere sein, da die Tinctur unter den günstigsten Bedingungen in obiger Beziehung endlich doch verdarb. Es blieb nur so viel ausgemacht, dass Licht und erhöhte Temperatur in der Tinc- 
tur schon vorhandene Disposition zum Verderben schneller entwickelten.

Janssen glaubte die Ursache dieser Zerselzung in einem Gehalte von essigsauren Alkalien in der Tinctur zu finden. Er meinte, dass das Eisenoxydhydrat, wenn man es mit ätzenden oder einfach - kohlensauren Alkalien fälle, einen namhaften Antheil des Fällungsmittels aufnehme, welcher beim Auflösen in Essigsäure in essigsaures Salz verwandelt werde, und bei der bekannten zersetzenden Einwirkung essigsaurer Alkalien auf die sogenannten auflöslichen basischen Eisenoxydsalze zum Verderben der essigsauren Eisentinctur Veranlassung gebe. Janssen will deshalb anderthalb- oder doppelt-kohlensaure Alkalien zur Bereitung des Eisenoxydhydrats verwandt wissen, weil er glaubt, dass alsdann jene Einmischung nicht statt finde. - Dass diese Ansichten manches Richtige enthielten, dass der von Janss en erwähnte Umstand namentlich an dem mit Gelatiniren verbundenen Verderben der Eisentinctur manchmal viel Antheil haben könne, liessen mich meine eigenen Erfahrungen ebenfalls nicht bezweifeln; aber ich erkannte doch auch, dass die obige Erklärung nicht für den ganzen Kreis der hieher gehörigen Erscheinungen passe, was unter anderm daraus hervorging, dass die nach der Janssen'schen Methode bereitele Tinctur sich ebenfalls, wenn auch langsam, zersetzte. Die Gegenwart essigsaurer Alkalien in der Tinctur schien mir ebenso, wie Licht und Wärme, mehr accessorisch bei der Zersetzung zu wirken.

Es wollte mir indessen lange nicht gelingen, andere Ursachen dieser Zersetzung aufzufinden, bis endlich einige Beobachtungen, die ich an einem in Breiform aufbewahrten Eisenoxydhydrat zu machen Gelegenheit fand, mich die Vorgänge allmälig richtiger verstehen liessen. Es ist schon von mehreren Seiten her hervorgehoben worden, dass das Eisenoxydhydrat unter solchen V̌mständen sehr bald auffallende Veränderungen erleidet. Unter diesen letzteren machte sich mir besonders auch das Unlöslichwerden desselben in Essigsäure bemerklich. Ein län- 
gere Zeit unter Wasser aufbewahrtes Hydrat verhielt sich in dieser Beziehung ganz, wie ein in der Wärme völlig ausgetrocknetes. Wie diese Erfahrung einerseits auf einen muthmaasslichen Zusammenhang der Auflöslichkeit des Oxyds mit einem bestimmten Wassergehalt in demselben hinwies, so konnte mir zugleich auch nicht die äussere Aehnlichkeit entgehen, welche die Umbildung des flüssigen Eisenoxydhydrats und die Zersetzung der essigsauren Eisenauflösung zeigten, und beides zusammen genommen liess mich die Frage aufwerfen, ob nicht etwa ein Hydrat von bestimmtem Wassergehalt in die Mischung des Liquor ferri acet. eingehe, und eben seiner Zersetzbarkeit wegen die erwähnten Erscheinungen bedinge. Diese Fragen haben eine Reihe von Beobachtungen und Versuchen hervorgerufen, deren Resultate ich in dem Nachfolgenden mittheilen will. Sie scheinen mir in obiger Beziehung wenig Zweifel übrig zu lassen, und werden daher auch uber die richtigste Art der Darstellung und Aulbewahrung des essigsauren Eisenliquors Aufschluss geben können. Ich wende mich zunächst zu den Eigenschaften und der $\mathrm{Zu}$ sammensetzung des Eisenoxydhydrats.

Das Eisenoxydhydrat, wie es aus den Auflösungen der Eisenoxydsalze oder des Eisenchlorids durch Fällung mit ätzenden oder kohlensauren Alkalien erhalten wird, bildet bekanntlich im noch feuchten Zustande eine braune oder gelbbraune, sehr hydratische, fast gallertartige Masse, welche beim Trocknen zu harten schwarzen, auf dem Bruche glasglänzenden Klumpen zusammengeht. Man hat darin 3 Atome Wasser angenommen, allein diese Annahme, die sich auf die Untersuchung eines völlig trocken gewordenen Niederschlages gründen mag, giebt den Wassergehalt des frisch gefallien noch feuchten Hydrats viel zu klein an; ein solches enthält wenigstens 6 , wahrscheinlich sogar 9 Atome Wasser. Als ich das mit überschiussigem Aetzammoniak gefällte und sorgfältig gewaschene Hydrat durch Pressen zwischen Löschpapier so weit getrocknet hatte, dass es sich, ohne merklich zusammen zu ballen, zum Pulver zerreiben liess, enthielt es 50 Proc. Wasser 
(durch Glihen bestimmi) also 9 Atome. Hier war natürlich auch etwas mechanisch anhängendes Wasser fortgegangen, aber es fragt sich, ob dieses Wasser nicht schon aus der Verbindung mit dem Eisenoxyde abgeschieden worden war; denn letzteres, obgleich noch völlig auflöslich in Essigsäure, erforderte von letzlerer doch schon eine ungleich grössere Menge. Wie zersetzbar aber das Hydrat sei, zeigte der fernere Verlauf des Versuches. Als ersteres nämlich zerrieben, und auf Papier ausgebreitet eine Nacht in gewöhnlicher Stubentemperatur gelegen hatte, enthielt es nur noch 37 Proc. Wasser. Es war nun staubig trocken geworden; diesem Unterschiede in dem äusseren Feuchtigkeitszustande während beider Tage entsprach aber die Verschiedenbeit des inneren Wassergehalts keineswegs, es musste nun jedenfalls Hydratwasser fortgegangen sein, und lässt sich also das wenigstens mit Sicherheit annehmen, dass der Wassergehalt des frisch gefallten Eisenoxydhydrats nicht weniger als 40 Proc. oder 6 Atome betrage - ln dem zuletzt erwähnten Zustande bildete das trockne Oxyd mit der Essigsäure zwar noch dunkelbraune Auflösungen, aber die Säuremenge, welche dazu erforderlich war, hatte sich sehr merklich erhöht.

Man sieht demnach schon, dass das Wasser in dem Eisenoxydhydrat nur äusserst schwach gebunden ist. Da dieses auf die Zerselzungs - Erscheinungen der essigsauren Eisentinctur vorzugsweise Licht wirft, so will ich daruber noch ferner Einiges aus meinen Versuchen anführen. Bei einer Wiederholung des oben erwähnten Versuchs betrug der Wassergehalt eines bis zur Zerreiblichkeit getrockneten Oxyds 46 Proc.; nach 24stundigem Trocknen auf Papier 33 Proc. Ein mit doppelt-kohlensaurem Natron gefälltes Oxyd zeigte unter gleichen Verhaltnissen 40 und 36 Proc. In allen Hydraten sank der Wassergehalt, wenn sie gepulvert der Luft ausgesetzt wurden, in wenigen Tagen auf 30 Proc. herab. In festen Klumpen hielten sie das Wasser lester gebunden, und ich fand in einem Falle nach 14 Tagen noch 36 - 38 Proc. In verschlossenen Gefässen über Schwefelsäure oder Chlorcalcium getrock- 
net, zeigle das Hydrat nach wenigen Tagen nur noch 25 bis 29 Proc., was dem Verhältnisse von 3 Atomen Wasser nahe kommt. Auch ein längere Zeit aufbewahrles in gewöhnlicher Temperatur getrocknetes Hydrat näherte sich in seinem Wassergeballe diesem Verhältnisse, und ich vermuthe, dass letzteres die Grenze bezeichnet, bis zu der die Zersetzung des Eisenoxydhydrats boi ge wöhnlich er Temperalur geht. Es kann daher nur die unter solchen Umständen erhaltene und völlig getrocknete Verbindung als $\mathrm{Fe}^{2} \mathrm{O}^{3}+3 \mathrm{Aq}$ gelten.

Bei Anwendung von Wärme geht jedoch die Zersetzung beträchtlich weiter. Im Wasserbade bei $+100^{\circ} \mathrm{C}$. kurze Zeit getrocknet, enthielt das Hydrat in mehreren Versuchen durchschnittlich 16 Proc., nach längerer Zeit 14-14: Proc., also elwa $1 \frac{1}{2}$ At. Wasser. In zwei anderen Versuchen erhielt ich nur 1.3 und $10 \frac{1}{2}$ Proc. Letztere Menge würde 1 At. Wasser entsprechen, und liegt vielleicht hier wiederum ein Grenzpunct, bis zu dem nämlich die Zersetzung des trockenen Hydrates bei einer höhern Temperatur, die jedoch nicht $100^{\circ} \mathrm{C}$. ubersteigt, gehen kann.

Für das Verständniss des Verhaltens der Tinct. ferri ac. ist nun aber besonders der Umstand von Wichtigkeit, dass dass Eisenoxydhydrat auch im feuchten Zustande mehr oder weniger schnell zersetzt wird. Mag man dasselbe in Breiform, oder in gepressten, noch feuchten Kuchen aufbewahren, in beiden Fällen nimmt es bald eine hellere Färbung an und wird, besonders im breiförmigen Zustande, allmälig hell ziegelroth. In einem Falle ging es sogleich in ein reines Ockergelb über. Zugleich erlangt das $\mathrm{Hy}$ drat eine mehr pulverige Beschaffenheit; dass es, wie Einige behauplen, krystallinisch werde, habe ich nicht wahrgenommen. Das gepresste Hydrat wird dabei feuchter und zerfliesst gewissermaassen. Trocknet man nun das so veränderte Hydrat an freier Luft oder über Schwefelsäure, so findet man, dass es den grössten Theil seines Wassers abgegeben hat. In meinen Versuchen zeigten mehrere Proben $11 \frac{1}{2}-12 \frac{1}{2}$ Proc. Glühverlust, was auf 
1 Atom Wasser in derselben schliessen lässt. Dass es dabei unlöslich in Essigsäure werde, wenigstens mit derselben nicht die bekannte dunkel gefärbte Auflösung bilde, habe ich schon erwähnt, und dass es sich ebenfalls nicht mehr mit arseniger Säure verbindet und in jenem $\mathrm{Zu}$ stande seine Eigenschaft als Gegengift gegen dieselbe gänzlich eingebuisst hat, werde ich weiter unten $z u$ besprechen haben.

Schneller noch geht auch hier die Zersetzung des Eisenoxydhydrats in höherer Temperatur vor sich. Erhitzt man frisch gefälltes Hydrat mit Wasser, so wird es sehr bald heller von Farbe und zugleich compacter, und löst sich dann ebenfalls nicht mehr in Essigsäure. In dem getrockneten Oxyde fand ich 7-8 Proc., in einigen Versuchen nur $4 \frac{1}{2}-5$ Proc. Wasser. Man wird letzteres ohno Zweifel ganz herauskochen können, und dass ein in der Siedhitze gefälltes Oxyd wenig oder gar kein Wasser enthalten werde, war darnach vorauszusehen; ich fand in der That in einem solchen Präparate nicht 3 Proc. flüchtiger Substanz.

Aus diesen Beobachtungen geht also, um es kurz zu wiederholen, mit Bestimmtheit hervor, theils dass das kalt gefällte Eisenoxydhydrat eine ungleich grössere Menge Wasser enthält, als man bisher geglaubt hat, und theils, dass dieses Wasser zum grössten Theile nur äusserst schwach gebunden ist, und sowohl durch geringe äussere Einflusse, als auch durch die Hinneigung des Eisenoxyds zu einer grösseren Cohärenz ausserordentlich leicht aus dem Hydrate abgeschieden wird. Sieht man nun die Zersetzungs - Erscheinungen, welche die essigsaure E isenauflösung zeigt und ihre Bedingungen näher an, und vergleicht sie mit denjenigen, die an dem in Wasser suspendirten Hydrat wahrgenommen werden, so kann man sich der Vermuthung nicht erwehren, dass hier sehr nahe Beziehungen obwalten. Dort wie hier entstehen allmalig Ausscheidungen, die sich in Essigsäure nicht, oder doch nur in einem bedeutenden Ueberschusse derselben auflösen. In beiden Fallen hat ferner die Temperatur auf 
die Schnelligkeit der Zersetzung gleichen Einfluss, denn bekanntlich wird die essigsaure Eisenauflösung beim Erhitzen augenblicklich zersetzt, und geht auch in mässig warmer Luft sebr bald dem Verderben entgegen. Gleiche Wirkungen lassen auf gleiche Ursachen schliessen. Wenn man sich die essigsaure Eisenauflösung, und iberhaupt alle sogenannten auflöslichen basischen Eisenoxydsalze, die einander in ihrer Bildungsweise, wie in ihren Eigenschaften sämmtlich sehr ähnlich sind, als Verbindungen von neutralen Salzen mil Eisenoxydhydrat denkt, und dabei berücksichtigt, dass diese Verbindungen nur sehr lose sein können, und dass das eine Glied derselben, das Eisenoxydhydrat, an sich sehr zersetzbar ist, so gewinnt die Leichtigkeit, mit der dieselben zerfallen, eine durchaus ungezwungene Erklärung. Der Annahme einer solchen Constitution dieser Eisensalze aber, die wir demnach gewissermaassen als Doppelsalze zu betrachten haben würden, in deren einem Gliede das Wasser die Säure repräsentirt, steht nicht allein nichts entgegen, sondern sie wird durch die Bildungsweise, so wie durch die Eigenschaften dieser Verbindungen, z. B. durch ihre Auflöslichkeit sehr unterstutzt. Wie gesagt, kann die Anziehung zwischen den näheren Bestandtheilen nur eine schwache sein, und daher wird das cohäsive Bestreben des Eisenoxyds in der Verbindung verhältnissmässig nur wenig vermindert sein; indessen schwächt die Verbindung dieses Bestreben allerdings, und es scheint, dass diese Wirkung durch eine möglichst niedrige Temperatur bedeutend unterstützt werde. $\mathrm{Ob}$ letzteres aber ganz bis zur Aufhebung jenes cohäsiven Bestrebens gehen könne und hiervon zugleich eine praktische Anwendung zu machen sei, darf noch nicht mit Bestimmtheit behauplet werden. Ich kann aus meinen bisherigen Erfahrungen nur das anfuihren, dass sich jede essigsaure Eisenauflösung, wie sie bereitet sein möge, in einer Temperatur über $+12^{\circ} \mathrm{C}$. früher oder später, gemeiniglich schon innerhalb 6 Monaten, zersetzt, dass mehrere vorsichtig bereitete Präparate dagegen in mit Löschpapier umwickelten Gefässen, die in ein flaches Gefäss 
mit Wasser gestellt und dadurch kühl erhalten, ausserdem aber an der kühlsten Stelle des Kellers aufbewahrt wurden, sich nunmehr fast ein ganzes Jahr völlig unverändert erhalten haben. Diese letztere Beobachtung dürfte für die Praxis weiter zu verfolgen sein.

Dass auch Lichteinwirkung die Zersetzung dieser Verbindung befördere, möchte ebenfalls nicht zu bezweifeln sein, und ist ein Schutz auch nach dieser Seite hin sehr anzuempfehlen. Den entschiedensten Einfluss in dieser Beziehung uben jedoch gewisse Beimengungen aus, die deshalb sorgfaltig gemieden sein wollen. Es ist schon hinreichend bekannt, dass die sogenannten auflöslichen basischen Eisenoxydsalze durch einen geringen Zusatz verschiedener Substanzen, z. B. von Salzen, namentlich von essigsauren Salzen, ja auch von Säuren augenblicklich zersetzt werden, besonders bei erhöhter Temperatur. Es sind dieses zum Theil sehr merkwïrdige, schwer zu verstehende Reactionen, uber die erst ein Zurückgehen auf die noch nicht genug studirten Eigenschaften der neutraJen Eisenoxydsalze Aufschlnss geben möchte. Das essigsaure Eisenoxydhydrat, wie ich unser Präparat der Kürze halber nennen will, ist in den erwähnten Beziehungen besonders empfindlich, und wohl deshalb, weil das neutrale Oxydsalz an sich schon sehr zersetzbar ist. Deshalb ist nun aber sicherlich auch die Bereitungsweise des Eisenoxydhydrats behuf der Darstellung der essigsauren Auflösung nicht gleichgültig, sofern dieses je nach dem angewandten Verfahren mehr oder weniger rein ausfallen kann. Diesen Einfluss hat schon Jans s en richtig erkannt, wie sich in dem Folgenden noch deutlicher herausstellen wird, wenngleich seine Schlussfolgerungen einer Berichtigung beduirfen möchten. Auch die Erfahrung von Walz, dass ein Kieselerdegehalt des Eisenoxydhydrats die Ursache der Zersetzung der essigsauren Auflösung sei, mag unter gleichen Beschränkungen hingenommen werden. In einer grossen Anzall von Versuchen, die ich hinsichlich der Haltbarkeit des nach verschiedener Weise dargestellten essigsauren Eisenoxydhydrats angestellt habe, ist von mir 
auch anf diese Umstände Rücksicht genommen worden, und mögen die Resultate hier in allgemeinen Umrissen Platz finden. Es wurden uberhaupt folgende Momente bei den Versuchen beachtet:

1) Zur Bereitung des Eisenoxydhydrats wurden die Lösungen von verschiedenen Eisenoxydsalzen, uämlich von schwefelsaurem und salpetersaurem Eisenoxyd, so wie die des Eisenchlorids verwandt.

2) Die Auflösungen wurden einmal im concentrirten Zustande ( 3 Unzen Flüssigkeit auf 40 Gran Eisen), das andere Mal verdünnt (24-48 Unzen Fluissigkeit auf 40 Gran Eisen) gefällt.

3) Als Fällungsmittel wurden verwandt: einfach-, anderthalb- und doppelt-kohlensaure Alkalien (meistens Natron-, in einigen Fällen auch Kalisalz); Aetzkali und Aetzammoniak. - Dass die Niederschläge stets mit grösster Sorgfalt gewaschen wurden, bedarf kaum einer Erwähnung. In den mit Aetzammoniak gefällten Niederschlägen habe ich alsdann keine Spur des Fällungsmittels wahrgenommen.

4) Das erhaltene Eisenoxydhydrat wurde in verschiedenen Feuchtigkeitzuständen geprüft. Die Entfernung des anhängenden Wassers geschah dabei stets durch Pressen des Hydrats zwischen Löschpapierlagen.

5) Zur Auflösung des Eisenoxydhydrats wurden Essigsäuren von verschiedener Stärke genommen; dieselbe wurde ubbrigens stets ohne alle Anwendung von Wärme bewirkt.

6) Die erhaltenen (filtrirten) Flüssigkeiten wurden unter verschiedenen Umständen in Beziehung auf Licht- und Wärme-Einwirkung aufbewahrt.

Die Ergebnisse dieser Versuche waren nun im Allgemeinen folgende:

1) Die Art des Eisensalzes zeigte im Ganzen nur einen sehr geringen Einfluss; doch trat letzterer in der Weise bemerkbar hervor, dass aus dem Eisenchlorid caeteris paribus stels die sich am schnellsten zersetzenden Auflösungen von essigsaurem Hydrat erhalıen worden. Um- 
gekehrt schien der grösste Vortheil hinsichtlich der Haltbarkeit sich auf die Seite des salpetersauren Eisenoxyds zu neigen.

2) Die Concentration der Eisenauflösungen hatte, wie es mir schien, einigen Eintluss auf die Auflöslichkeil des Hydrats, und zwar so, dass das aus concentrirten Auflösungen erhaltene etwas mehr Säure erforderte, als das mit verdiunnteren dargestellte. Jedoch habe ich dieses nur bei Anwendung von ätzenden Alkalien bemerken können. In der Haltbarkeit des ferligen Präparats war kein Unterschied wahrzunehmen.

3) Durch Fällung der Auflösungen mit einfach - kohlensauren Alkalien wurde unter allen Umständen ein leicht auflösliches Hydrat erhalten, welches jedoch alle Male eine wenig hallbare, meistens sehr bald gelatinirende Auflösung gab.

4) Anderthalb- und doppelt-kohlensaure Alkalien lieferten ein vorzugsweise leicht auflösliches Hydrat. Die aus 1 Th. Eisen erhaltene Menge liess sich so ziemlich in $3 \mathrm{Th}$. Essigsäure von 1,040-1,045 auflösen. Die dadurch gebildeten Flüssigkeiten gelatinirten jedoch $\mathrm{s} \ddot{\mathrm{m}} \mathrm{mt}$ Jich, zwar nicht sogleich, aber doch innerhalb 6 Monaten. Waren sie aus Eisenchlorid erhalten, so trübten sie sich zugleich, während die aus schwefelsaurem und salpetersaurem Eisenoxyd dargestellten Präparate eine fast ganz klare Gallerte bildeten.

5) Die ätzenden Alkalien, Kali und Ammoniak, lieferten übereinstimmend ein Eisenoxydhydrat, welches beträchtlich grössere Säuremengen zur Aullösung erforderte, als das mit kohlensauren Alkalien erhaltene. Das geringste Verhältniss war 4 Th. Essigsäure von 1,045 auf 1 Th. Eisen. Daneben zeigte die Concentration der Eisenauflosung, aus der das Hydrat gefallt worden war, und besonders auch der Grad der Trockenheit einen merklichen Einfluss auf die Löslichkeil des Hydrats. Liess man die Presse so lange einwirken, dass das Gewicht des Hydrats weniger als das Sechsfache vom angewandien Eisen betrug, so stieg die Menge der zur Aufösung erforderlichen Essig- 
säure auf 6 Theile. Sämmtliche Auflösungeu haben sich seit ibrer Darstellung ( $10-12$ Monate) unverändert aufbewahren lassen, wobei sie jedoch in der erwähnten Weise fortwährend kühl gehalten worden sind.

6) Eine Essigsäure von 1,065 spec. Gew. lieferte Auflösungen, die offenbar weniger haltbar waren, als die mit der erwähnten schwächeren Säure erhaltenen. Es zeigte sich zwar kein Gelatiniren der Flüssigkeiten, wohl aber eine successive Ablagerung eines pulverigen Niederschlages. - Diese Beobachtung lässt fast vermuthen, dass auch eine zu grosse Concentration des essigsauren Eeisenoxydhydrats seine Haltbarkeit beeinträchtigt, und gewiss ist jedenfalls, dass sie dieselbe nicht vermehrt.

Den Einfluss des Lichts und der Temperatur auf die Haltbarkeit des Präparates habe ich bereits oben berührt. lch erwähne nur noch, dass ich in den Bodensätzen der zersetzten Auflösungen Kieselerde in merklicher Menge nicht wahrgenommen habe, und letztere daher auch nicht als eine allgemeine Ursache des Verderbens ansehen kann.

Das aus diesen Beobachtungen für die Darstellungsweise eines möglichst halıbaren, essigsauren Eisenoxydhydrats abzuleitende praktische Resultat lässt sich mit Wenigem zusammenfassen. Zunächst empfiehlt sich das salpetersaure Eisenoxyd als das beste Mlaterial zur Darstellung eines möglichst reinen Eisenoxydhydrats. Um aus der Auflösung des Eisens in überschüssiger Salpetersäure einen etwaigen Kieselerdegehalt zu entfernen, wird es gerathen sein, erstere bis ganz zur Trockenheit zu verdampfen, und den Rückstand in angesäuertem Wasser wieder aufzunehmen.

Das Eisenoxydhydrat muss aus der stark verdünnten Auflösung (48 Th. Wasser auf $1 \mathrm{Th}$. Eisen) mittelst raschen Zusatzes des Fällungsmittels gefailt werden. Man bedient sich dazu des Aetzammoniaks, da Aetzkali theurer und gewöhnlich kieselerdehaltig ist, und übrigens keine Vorzuige besitzt. Auf $1 \mathrm{Th}$. Eisen werden etwa $9 \mathrm{Th}$. Aetzammoniak von 0,96 spec. Gew., um letzteres überschiissig zu haben, erforderlich sein. Mehr noch möchte sich das 
Geiseler'sche Verfahren empfehlen, nämlich die auf $18 \mathrm{Th}$. verdünnte Eisenauflösung in die mil 60 Unzen Wasser vermischte Ammoniakfluissigkeit zu giessen, um so die Einmischung von basischem Salz und Ammoniak möglichst zu verhindern. - Das erhaltene Eisenoxydhydrat muss auf das sorgfältigste ausgewaschen werden. Dass Fällen und Auswaschen durchaus kalt geschehen müsse, versteht sich bei der grossen Zersetzbarkeit des Hydrats von selbst.

Der auf dem Filter befindliche Niederschlag wird zwischen oft zu erneuernden Lagen von Löschpapier so lange gepresst bis das Gewicht desselben das Achtfache vom angewandten Eisen beträgt, was sich annahernd sehr wohl bestimmen lässt, und hierauf mit \& Unzen Essigsäure von 1,040-1,040̈ spec. Gew. übergossen. Die Autösung wird binnen einigen Tagen vollständig oder fast vollständig erfolgen. Man darf sie keinenfalls durch Anwendung von Wärme beschleunigen wollen; im Gegentheil muss man das Gefäss schon jetzt kühl halten. Die gebildete Fluissigkeit lässt man während einiger Tage sich absetzen, giesst sie klar von dem Bodensatze ab, filtrirt den Rest und bewahrt das Präparat, aus welchem jederzeit die Tinct. ferri acetic. aeth. gemischt werden kann, in wohl verschlossenen, mit Löschpapier umwickelten, und in ein flaches Gefäss mil Wasser gestellten Flaschen an einem möglichst kühlen und zugleich dunkeln Orte auf.

Was die eben gegebene Gewichtsbestimmung anlangt, so muss ich darüber noch einige Bemerkungen hinzufügen, in denen ich veranlasst sein werde, auf die Zusammensetzung des essigsauren Eisenoxydhydrats in quantitativer Beziehung, so wie auf den muthmaasslichen $\mathrm{Zu}$ sammenhang einiger der oben erwähnten Erscheinungen zuruickzukommen. Es ist durchaus erforderlich, bei der Darstellung unseres Präparates bestimmte quantilative Normen zu befolgen, indem die Beschaffenheit desselben andernfalls sehr verschieden ausfallen müsste, theils hinsichtlich des absoluten Eisengehaltes, theils und zwar vorzuiglich hinsichtlich der Verbindungsweise des Eisens,

Arch. d. Pharm. CIX. Bds. 3. Hft. 
die mir hier keineswegs gleichgültig zu sein scheint. Ich habe oben angefuhrt, dass das Eisenoxydhydrat sich sehr verschieden auflöslich in Essigsäure zeigt, je nachdem es mehr oder weniger durch Pressen ausgetrocknet worden ist. Geiseler, der diesen Umstand noch genauer ins Auge gefasst hat, fand ibereinstimmend mit den angefuihrten Beobachtungen, dass ein Hydrat, welches auf ungefähr $3 \frac{1}{2}$ Th. von $1 \mathrm{Th}$. Eisen abgepresst worden war, doppelt soviel Essigsäure zur Aullösung verlangte, als ein nur auf $7 \mathrm{Th}$. ausgetrocknetes. $\mathrm{Da}$ es in beiden Fällen jedoch noch feucht geblieben war, so darf aus jenem Unterschiede: in der Auflöslichkeit mit Recht wohl auf eine in der Constitution des Hydrals vorgegangene Veränderung geschlossen werden. Nach meinen oben mitgetheilten Untersuchungen über den Wassergehalt des Eisenoxydhydrats in seinen verschiedenen Zuständen kann es aber im ersten Falle schon nicht weniger als 6 At. Wasser enthalten haben, und in dem letzten von Geiseler angeführten Falle musste der Wassergehalt also noch beträchtlich grösser gewesen sein. Ich werde dadurch noch mehr in der schon oben ausgesprochenen Vermuthung bestärkt, dass das frisch gefällte Eisenoxydhydrat wirklich mindestens 9 At. Wasser enthalte. Ferner erwähnte ich oben, dass ein mit doppelt-kohlensaurem Natron gefalltes und nicht zu stark ausgepresstes Hydrat nur $3 \mathrm{Th}$. Essigsäure von 1,040 spec. Gew. (auf $1 \mathrm{Th}$. Metall berechnet) zur Auflösung erfordert habe. Damit ziemlich übereinstimmend giebt Janssen die für eine gleiche Menge noch schwächer gepressten Hydrats erforderliche Säuremenge vorı $1,040-1,045$ auf $2 \frac{1}{4} \mathrm{Th}$. an. Berechnet man nun aus dem Durchschnitte dieser Mengen das Atomverhältniss von Eisenoxyd und Essigsäure in der Verbindung, so findel man, dass es sich annähernd verhält, wie $1: \frac{3}{4}$ oder wie $4: 3$. Demnach würde ich für die aus schwach gepresstem Eisenoxydhydrat und Essigsäure gebildete Verbindung; nach meiner Betrachtungsweise die Formel construiren:

$$
\left(\mathrm{Fe}^{2} \mathrm{O}^{3}+3 \overline{\mathrm{A}}\right)+3\left(\mathrm{Fe}^{2} \mathrm{O}^{3}+9 \mathrm{Aq}\right) \text {. }
$$

Geiseler fand ferner noch, dass ein bis zum Zer- 
reiblichwerden ausgetrocknetes Hydrat so viel Säure zur Auflösung erfordere, als zur Bildung des neutralen (dreifachsauren) essigsauren Eisenoxyds nölhig sei, dass also ein solches Hydrat nur das letztere Salz bilde. Zieht man meine mehrfach angefuhrte Erfahrung, dass das so weit ausgetrocknete Hydrat aber immer noch mindestens 6 At. Wasser enthalten muisse, hinzu, so muss - beide Beohachtungen als richtig angenommen - gefolgert werden, dass nur das Ifydrat $\mathrm{Fe}^{2} \mathrm{O}^{3}+9 \mathrm{Aq}$ mit dem neutralen essigsauren Eisenoxyd eine Verbindung einzugehen vermag, und dass, wenn wirklich ein $\mathrm{Fe}^{2} \mathrm{O}^{3}+6 \mathrm{Aq}$ existirt, woran ich kaum zweifle, dieses eben sowohl, wie die Verbindung $\mathrm{Fe}^{2} \mathrm{O}^{3}+3 \mathrm{Aq}$ beim Uebergiessen mit Essigsäure in Eisenoxyd und Wasser zerfalle, und demnach nur neutrales Salz bilde. Unter diesem Gesichtspuncte bört die Verschiedenheit der Säuremengen, welche zum Auflösen des mehr oder minder gepressten Hydrats erforderlich sind, auf, ein Räthsel zu sein. Die Verbindung $\mathrm{Fe}^{2} \mathrm{O}^{3}+$ $9 \mathrm{Aq}$ ist, wie sich hinreichend ergeben hat, äusserst zersetzbar und giebt schon beim Auspressen einen Theil ihres Wassers ab. Presst man daher nur wenig, so wird ganz oder grösstentheils $\left(\mathrm{Fe}^{2} \mathrm{O}^{3}+3 \overline{\mathrm{A}}\right)+3\left(\mathrm{Fe}^{2} \mathrm{O}^{3}+\right.$ $9 \mathrm{Aq})$ gebildet; geht aber die Entwässerung weiter, so wird ein Theil des neunfach gewässerten Hydrats in sechs- oder gar in dreifach gewässertes umgeändert, welche letztere beiden Verbindungen mit der Essigsäure nur neutrales Salz bilden, und daher grössere Mengen derselben zur Auflösung erfordern.

Hiermit ist auch die grosse Verschiedenheit der Angaben von Janssen, Wittstein, Bette, Duflos, Trautwein, Sch lick um und mehrerer neuerer Pharmakopöen über die zu befolgende quantilative Norm genügend erklärt. In diesen Angaben durchläuft das Verhälıniss, zu welchem das Hydrat, auf 1 Theil des angewandten Eisens berechnet, durch Auspressen gebracht werden soll, die Zahlen $3 \frac{1}{2}$ bis 10. Als zum Aullösen des Hydrats erforderliche Säure (von 1,040-1,045 spec. Gew.) werden $2 \frac{1}{4}-10$ Theile, und von Schlickum sogar doppelt so 
viel, als zur Bildung von. neutralem Salze erforderlich ist, nämlich $24 \mathrm{Th}$. angegeben! Es thut wahrlich Noth, diesem Wirrwarr, bei dem der Mangel einer allgemeinen deutschen Pharmakopöe einmal wieder recht fühlbar wird, ein Ende zu machen. - Es erklärt sich aus der obigen Annahme ferner noch die auffallende Löslichkeit des mit doppelt- und anderthalb-kohlensaurem Alkali gefältten Hydrates. Ein solches unterscheidet sich auch schon äusserlich durch seine zimmigelbe Farbe und die mehr pulverige Beschaffenheit von dem mil ätzenden Alkalien dargestellten. Es brauset selbst im gepressten Zustande ziemlich stark mit Säuren; und ich vermuthe deshalb, dass es eine Verbindung von Eisenoxydhydrat mit kohlensaurem Eisenoxyd sei, in welcher ersteres mehr vor Zersetzung geschützt ist, so dass es sich beim Austrocknen länger auflüslich erhält, als das mit ätzenden Alkalien niedergeschlagene reine Hydrat.

Der procentische Eisengebalt kann in den auf verschiedene Weise bereiteten Auflösungen des Eisenoxydhydrates bei nichı gar zu sehr abweichenden Verhältnissen ziemlich gleich sein, indem sicb, wie auch Geiseler gezeigt hat, die Abnahme des Wassers im Hydrat mit der Zunahme der Säure im Gewichte ausgleicht; allein die Verbindungen können nichts desto weniger sehr von einander verschieden sein, und zwar darin, dass sie in den Verhältnisse ihrer näheren Bestandtheile, nämlich des neutralen essigsauren Eisenoxydes zum Eisenoxydhydrat abweichen. Dieses ist meines Erachtens durchaus nicht gleichgültig, und macht vorzugsweise eine quantitative Norm erforderlich. Es fragt sich nur, wie hier der richtigste Maassstab zu finden sei. Die Verbindung $\left(\mathrm{Fe}^{2} \mathrm{O}^{3}\right.$ $+3 \overline{\mathrm{A}})+3\left(\mathrm{Fe}^{2} \mathrm{O}^{3}+9 \mathrm{Aq}\right)$, wie man sie mit sehr schwach gepresstem Eisenoxydhydrat erhält, würde sich aus mehreren Rücksichten und namentlich als das gleicbförmigste Präparat empfehlen; aber es scheint, als sei dieselbe zu sebr der Zersetzung, mindestens dem Gelatiniren unterworfen, und müsse deshalb das Verhältniss des neutralen Salzes etwas vergrössert werden. In dem Präparate der 
preussischen Pharmakopöe scheint mir dagegen die Menge des neutralen Salzes wiederum zu gross zu sein, indem darin 2 At. des letzteren nur mit 1 At. des $\mathrm{Fe}^{2} \mathrm{O}^{3}+9 \mathrm{Aq}$ verbunden sind. Ich glaube deshalb, dass man einen Mittelweg einschlagen müsse, und habe zu dem Ende in meiner obigen Vorschrift angegeben, das von $1 \mathrm{Th}$. Eisen erhaltene Hydrat auf $8 \mathrm{Th}$. abpressen zu lassen, und es in 4 Th. Essigsäure von 1,040 spec. Gew. aufzulösen. Diese Verbindung, die richtig bereitet haltbar $z \mathbf{u}$ sein scheint, enthält auf 3 At. Eisenoxyd 4 At. Essigsäure; oder 2 At. $\mathrm{FeO}^{3}+3 \overline{\mathrm{A}}$ sind darin mit $2 \frac{1}{2}$ bis $3 \mathrm{At} . \mathrm{Fe}^{2} \mathrm{O}^{3}+9 \mathrm{Aq}$ vereinigt.

Dass man aber in das Präparat so viel Eisenoxydhydrat bringe, als sich mit der Haltbarkeit desselben nur immer verträgt, möchte aus mehr als einer Rücksicht rathsam sein. Einmal scheint mir die medicinische Wirksamkeit des Mittels, die Leichtigkeit, mit der dasselbe vertragen wird, zum Theil von dem Gehalt an Eisenoxydhydrat abzuhängen. Wäre dem nicht so, so würde man nur das ungleich haltharere neutrale Salz darzustellen brauchen. Sodann aber habe ich auch die Anwendung des essigsauren Eisenoxydhydrates als Gegengift gegen arsenige Säure im Auge. Das Eisenoxydhydrat für sich, so wirksam es in dieser Bezielung frisch bereitet obne Zweifel ist, so unzuverlässig wird es nach und nach beim Aufbewahren. Es wirkt nur mil seinem vollen Wassergehalt, und die Ursache liegt vermuthlich darin, dass, wie auch Berthold und Bunsen vermuthen, das Hydrat nur als solches in die Verbindung mit arseniger Säure, oder vielmehr mit arsenigsaurem Eisenoxyd übergeht, und damit das bekannte unauflösliche Salz bildet. Das Eisenoxydhydrat verliert aber, wie ich oben erwähnt habe, beim Aufbewahren unter Wasser seinen Wassergehalt ganz oder theilweise, und kann deshalb nicht wirken. Ich habe mich auch durch Versuche auf das bestimmteste überzeugt, dass ein längere Zeit aufbewahrtes Eisenoxydhydrat nur äusserst wenig arsenige Säure aufnimmt. Diese Erfahrung, die ja bereits auch von Anderen gemacht und hervor- 
gehoben worden ist, würde dem essigsauren Eisenoxydhydrat mit möglichst hohem Gehalt an Hydrat, welches als Antidot gegen arsenige Säure dem reinen Eisenoxydhydrat gleich wirkend angesohen werden darf, eine doppelte Wichtigkeit verleihen, sobald es gelänge, dasselbe haltbar herzustellen. Dieses möge demnach, und abgesehen von der medicinischen Wirksamkeit des Präparats. für die Praktiker ein Beweggrund sein, auf eine vervollkommnete Darstellungsweise ihr ferneres Augenmerk zu richten, und zu dem Ende auch meine obigen Angaben und Vorschläge der Prüfung zu unterwerfen.

$\mathrm{Nach}$ sch rift. Nach dem Schlusse der obigen Arbeit kommen mir noch zwei Abhandlungen über denselben Gegenstand zu Gesichte, die ich nicht unerwähnt lassen darf. Die eine derselben, welche in dem letzten Jahrgange des Buchner'schen Repertoriums enthalten ist, ist von Wittstein. Sie enthält für mich keine neuen Gesichtspuncte. Der Verfasser, der die Janssen'sche Arbeit offenbar nicht gekannt hat, stellt darin die Ansicht auf. dass die Zersetzbarkeit des Präparats in einer Neigung desselben, noch basischere Producte zu bilden, begründet sei. Dass etwas Derartiges eine mitwirkende Ursache sein könne, will ich gern zugeben; dass aber darin nicht die Hauptursache der Erscheinung liegen könne, werde jch hier nicht mehr zu erörtern brauchen.

Die zweite Arbeit ist die bereits vor mehreren Jahren erschienene Abhandlung T rau t we in's uber diesen Gegenstand. Ich kannte dieselbe bisher nur aus einer unbedeutenden Notiz im pharmaceutischen Centralblatte, ersehe nun aber, dass Traulwein bereits damals im Wesentlichen ganz dieselben Ansichten bezüglich der chemischen Constitution des Liquor ferri acet. ausgesprochen hat, als sie von mir oben entwickelt worden sind $\left.{ }^{*}\right)$. Ich war ver-

*) Mein werther Freund, Hr. Apotheker Sonneborn in Delbrück, macht mich derauf aufmerksam, dass auch $H$. Ro se schon vor mehreren Jahren in seinen Vorlesungen über organische Chemie ähnliche Ansichten vorgetragen habe. 
pflichtet, dieses hier noch zu bemerken, und darf wohl hinzufügen, dass ich sehr erfreut bin, mit einem so ausgezeichneten Pharmaceuten, hinsichtlich des vorliegenden Gegenstandes, in einigen Beziehungen zu gleichen Resultaten gelangt zu sein.

\section{Untersuchung eines Harnsteines;}

von

\section{Dr. Bernhard Sthamer.}

Carl P., Arbeitsmann, 32 Jahre alt, von schmächtigem Körperbau, seit Johannis 1847 an Urinbeschwerden leidend, ward im Mai v. J. in das híesige (Rostock) allgemeine Krankenhaus aufgenommen, und bei der mit ihm angestellten Untersuchung die Gegenwart eines Entenei-grossen Steines in der Blase entdeckt.

Der Patient war blass, abgemagert und entkräftet; der Urin lloss fortwährend tropfenweise, ungefähr in einer Quantität von 4-5 Pfund pro Tag ab. Letzlerer, von blassgelber Farbe, war frisch gelassen trüb, reagirte neutral, selten schwach sauer, ward aber bald alkalisch und bildete schon nach kurzem Stehen ein reichliches, schweres, weisses Sediment; spec. Gew. $=1,011-1,017$. Die festen Bestandtheile, namentlich die Salze, zeigten sich bedeutend vermindert, ebenso der Harnstoff. . Vom Sediment abfiltrirt, liess sich in der abfiltrirten Flüssigkeit durch Erhitzen ein geringer Gehalt an Albumin nachweisen.

Das Sediment bestand aus einer zusammenhängenden gelatinösen Masse, die unter dem Mikroskop zahlreiche Eiterkörperchen und Krystalle von phosphorsaurer Ammoniakmagnesia erkennen liess.

Bei der durch Hrn. Ober-Med.-Rath Prof. Dr. Strempel ausgefubrten Operation (Lateralschnill) wurde ein Stein von der oben angegebenen Grösse vorgefunden und seiner Grösse wegen in Fragmenten herausgefördert. Das 